\title{
EIGENFREQUENCIES AND EXPANSIONS FOR DAMPED WAVE EQUATIONS *
}

\author{
MICHAEL HITRIK ${ }^{\dagger}$
}

\begin{abstract}
We study eigenfrequencies and propagator expansions for damped wave equations on compact manifolds. Under the assumption of geometric control, the propagator is shown to admit an expansion in terms of finitely many eigenmodes near the real axis, with an error term exponentially decaying in time. In the presence of a nondegenerate elliptic closed geodesic not meeting the support of the damping coefficient, we show that there exists a sequence of eigenfrequencies converging rapidly to the real axis. In the case of Zoll manifolds, we show that the propagator can be expanded in terms of the clusters of the eigenfrequencies in the entire spectral band.
\end{abstract}

1. Introduction and statement of results. In this paper we shall study some problems arising in the spectral analysis of dissipative wave equations on compact manifolds. In order to formulate the results, we shall begin by recalling some standard assumptions and hypotheses.

Let $M$ be a compact connected smooth Riemannian manifold of dimension $\geq 2$, and let $\Delta$ be the corresponding Laplace-Beltrami operator. We consider the Cauchy problem for the wave equation with a damping term,

$$
\left\{\begin{array}{l}
\left(-D_{t}^{2}-\Delta+2 i a(x) D_{t}\right) u=0, \quad(t, x) \in \mathbf{R}_{+} \times M \\
\left.u\right|_{t=0}=u_{0} \in H^{1}(M),\left.\quad D_{t} u\right|_{t=0}=u_{1} \in L^{2}(M)
\end{array}\right.
$$

Here $D_{t}=\partial_{t} / i$, and the damping coefficient $a$ is a bounded nonnegative function on $M$, which is not identically zero. For simplicity, we shall assume that $a \in C^{\infty}\left(M ; \overline{\mathbf{R}_{+}}\right)$. The motivation for studying this problem comes from the geometric control theory (see [16] and [2]), where one is interested in the long time behaviour of solutions to (1.1), in relation to the geometry of the underlying manifold and the control (damping) region.

Associated with the evolution problem (1.1) is the solution operator $\mathcal{U}(t)=e^{i t \mathcal{A}}$, $t \geq 0$, acting in the Hilbert space of the Cauchy data $\mathcal{H}=H^{1} \times L^{2}$ and mapping $\left(u_{0}, u_{1}\right) \in \mathcal{H}$ to $\left(u(t, \cdot), D_{t} u(t, \cdot)\right)$. Here we equip $\mathcal{H}$ with the norm

$$
\left\|\left(\begin{array}{l}
u_{0} \\
u_{1}
\end{array}\right)\right\|_{\mathcal{H}}^{2}=\left\|\nabla u_{0}\right\|_{L^{2}}^{2}+\left\|u_{0}\right\|_{L^{2}}^{2}+\left\|u_{1}\right\|_{L^{2}}^{2} .
$$

The semigroup $\mathcal{U}(t)$ can be introduced by means of the Hille-Yosida theorem - see Section 2 for the corresponding resolvent estimates. The infinitesimal generator $\mathcal{A}$ is the operator

$$
\mathcal{A}=\left(\begin{array}{cc}
0 & 1 \\
-\Delta & 2 i a(x)
\end{array}\right): \mathcal{H} \rightarrow \mathcal{H}
$$

with the domain $D(\mathcal{A})=H^{2} \times H^{1}$. Here $H^{s}=H^{s}(M)$ is the standard Sobolev space on $M$. It follows that the spectrum of $\mathcal{A}, \operatorname{Spec}(\mathcal{A})$, is discrete.

The energy of the solution $u(x, t)$ of $(1.1)$ at time $t$,

$$
E(u, t)=\frac{1}{2} \int_{M}\left(|\nabla u|^{2}+\left|D_{t} u\right|^{2}\right) d x
$$

\footnotetext{
${ }^{*}$ Received October 7, 2003; accepted for publication December 22, 2003.

${ }^{\dagger}$ Department of Mathematics, University of California, Los Angeles, CA 90095-1555, USA (hitrik@math.ucla.edu).
} 
is nonincreasing as $t \rightarrow \infty$, and relations between the rate of the decay of the energy and the spectrum of $\mathcal{A}$ were studied in [16] and [20]; below we shall recall some of these results. The asymptotic distribution of the eigenvalues of $\mathcal{A}$ has been studied by Sjöstrand [22], and some further results in the case when the geodesic flow on $M$ is periodic have been obtained in [12]. We also remark that under the assumption of periodicity of the flow, much more precise results are possible in the two-dimensional analytic case, and we refer to [14], as well as to forthcoming papers in this series, for a detailed study of this problem. In the present work we shall be concerned with results, valid in all dimensions $\geq 2$.

We notice that $\tau \in \mathbf{C}$ is an eigenvalue of $\mathcal{A}$ precisely when the equation

$$
\left(-\Delta+2 i \tau a(x)-\tau^{2}\right) u(x)=0
$$

has a nonvanishing smooth solution. The eigenvalues $\tau$ will also be called the eigenfrequencies. The multiplicity $m\left(\tau_{0}\right) \in\{1,2 \ldots\}$ of an eigenfrequency $\tau_{0}$ is defined as the rank of the spectral projection

$$
\Pi_{\tau_{0}}=\frac{1}{2 \pi i} \int_{\gamma}(\tau-\mathcal{A})^{-1} d \tau,
$$

where $\gamma$ is a sufficiently small circle centered at $\tau_{0}$. We shall let $E_{\tau_{0}}:=\Pi_{\tau_{0}} \mathcal{H}$ stand for the generalized eigenspace corresponding to $\tau_{0}$.

It follows easily from (1.3) that if $\tau$ is an eigenfrequency, then

$$
0 \leq \operatorname{Im} \tau \leq 2\|a\|_{\infty},
$$

and since (1.3) is invariant under the map $(\tau, u) \rightarrow(-\bar{\tau}, \bar{u})$, the set of the eigenfrequencies is symmetric with respect to the reflection in the imaginary axis. We remark that $\tau=0$ is an eigenfrequency corresponding to the constant solution of (1.3), and in [16] it was proved that $\tau=0$ is the only eigenfrequency with the vanishing imaginary part.

Let us introduce a strongly continuous family of operators

$$
U(t): L^{2} \rightarrow H^{1}, \quad t \geq 0,
$$

which takes a vector $f \in L^{2}$ to the first component of $\mathcal{U}(t)$ applied to a vector of the form $(0, f)$. The mapping in (1.4) will be referred to as the propagator. A simple computation shows that in terms of $U(t)$, the matrix representation of the semigroup $\mathcal{U}(t)$ is given by

$$
\mathcal{U}(t)=\left(\begin{array}{cc}
V(t) & U(t) \\
D_{t} V(t) & D_{t} U(t)
\end{array}\right): \mathcal{H} \rightarrow \mathcal{H},
$$

where $V(t) f=D_{t} U(t) f-2 i U(t)(a f)$.

In the self-adjoint case, i.e. when $a \equiv 0$, it is true that $U(t)=i \sin t \sqrt{-\Delta} / \sqrt{-\Delta}$, and we have a Fourier expansion,

$$
\frac{\sin t \sqrt{-\Delta}}{\sqrt{-\Delta}} f(x)=\sum_{\lambda_{j}^{2} \in \operatorname{Spec}(-\Delta)} \frac{\sin \lambda_{j} t}{\lambda_{j}} \varphi_{j}(x),-\Delta \varphi_{j}(x)=\lambda_{j}^{2} \varphi_{j}(x),
$$

where the convergence is absolute in the case of smooth data. In this paper, we shall be interested in eigenfunction expansions of the propagator $U(t)$, as $t \rightarrow \infty$, in the 
case of the nonvanishing damping term. We are therefore concerned with relations between the long time behaviour of $\mathcal{U}(t)$ and the spectrum of $\mathcal{A}$. This problem is closely related to that of completeness and summability of generalized eigenfunction expansions of the operator $\mathcal{A}$. The classical theory of non-selfadjoint operators, as described in [11] and [17], shows that the space of finite linear combinations of the generalized eigenfunctions of $\mathcal{A}, \bigoplus_{\tau \in \operatorname{Spec}(\mathcal{A})} E_{\tau}$, is dense in $\mathcal{H}$. However, as emphasized in [1], the lack of orthogonality in the direct sum above indicates that the generalized eigenfunctions may still fail to form a (Riesz) basis in $\mathcal{H}$. The question of basisness in the context of weak abstract non-selfadjoint perturbations of selfadjoint operators has been studied in [17], and when specialized to the case at hand, the results of [17] apply in dimension one only, implying then the existence of a Riesz basis of the generalized eigenfunctions. See also [8] and [21]. Here we shall be concerned with the case $n \geq 2$, and as well as using the classical non-selfadjoint theory, we shall also rely on direct microlocal methods.

In addition to [17], in this paper we have been motivated by recent studies of expansions for propagators on unbounded domains in terms of the resonance statessee [28] for an overview of this work and further references. Our Theorems 1.2 and 1.3 below complement the corresponding results in the theory of resonances in a situation, when the decay of states is caused by a direct dissipative mechanism on a compact manifold. In Theorem 1.4 we state a result which, to the best of our knowledge, has no analogue for problems on unbounded domains.

When $p(x, \xi)=\xi^{2}$ is the principal symbol of $-\Delta$ defined on $T^{*} M$, we introduce the Hamilton vector field $H_{p}$, and recall that the integral curves of the Hamilton flow $\exp \left(t H_{p}\right): p^{-1}(1) \rightarrow p^{-1}(1)$ are mapped via the projection $\pi: T^{*} M \rightarrow M$ to geodesics on $M$. When $T>0$, we put

$$
\langle a\rangle_{T}=\frac{1}{T} \int_{0}^{T} a\left(\exp \left(t H_{p}\right) d t \text { on } p^{-1}(1) .\right.
$$

We define also

$$
0 \leq A(T)=\inf _{p^{-1}(1)}\langle a\rangle_{T},
$$

which is a bounded continuous function on $\mathbf{R}_{+}$. We recall from [16] that the limit

$$
A(\infty):=\lim _{T \rightarrow \infty} A(T)
$$

exists and it is true that $A(\infty)=\sup _{T>0} A(T)$.

In what follows we shall say that the geometric control condition holds if there exists a time $T_{0}>0$ such that any geodesic of length $\geq T_{0}$ meets the open set $\{x ; a(x)>0\}$. In this case it has been established by J. Rauch and M. Taylor [20] that there is a uniform exponential decay of the energy $E(u, t)$. A proof of this statement can also be extracted from the arguments given in Section 3 below.

Our starting point is the following result, which is essentially due to [16], and was also proved in [22].

THEOREM 1.1. Assume that the geometric control condition holds. Then $A(\infty)>$ 0 , and, for every $\varepsilon \in(0, A(\infty))$, there are at most finitely many eigenfrequencies in the strip

$$
\mathbf{R}+i[0, A(\infty)-\varepsilon]
$$


Our first result gives an expansion of the propagator $U(t)$, as $t \rightarrow \infty$, in terms of the eigenfrequencies in the strip (1.7), provided that the geometric control condition is satisfied. This results is analogous to the corresponding one in scattering theory, giving an expansion of the wave group in the exterior of a nontrapping obstacle, in terms of exponentially decaying resonant modes. See [26] and references given there.

THEOREM 1.2. Assume that the geometric control condition holds. Let $\varepsilon \in$ $(0, A(\infty))$ be such that there are no eigenfrequencies with the imaginary part $=A(\infty)-$ $\varepsilon$. Then there exists $C=C(\varepsilon)>0$ such that

$$
U(t)=\sum_{\tau \in \operatorname{Spec}(\mathcal{A}), \operatorname{Im} \tau<A(\infty)-\varepsilon} e^{i t \tau} p_{\tau}(t)+E(t), \quad t>0,
$$

where $p_{\tau}(t)$ is a polynomial in $t$ with values in $\mathcal{L}\left(L^{2}, H^{s}\right)$ for any $s \in \mathbf{R}$, and the remainder term satisfies

$$
\|E(t)\|_{\mathcal{L}\left(L^{2}, L^{2}\right)} \leq C e^{-(A(\infty)-\varepsilon) t}
$$

We have

$$
p_{\tau=0}(t) f=\frac{i \int f(x) d x}{\int 2 a(x) d x}, \quad f \in L^{2} .
$$

In order to motivate the next result, let us recall that in [16], G. Lebeau determined the optimal rate of the exponential decay of the energy $E(u, t)$. See also [20]. Set

$$
D=\inf \{\operatorname{Im} \tau ; \tau \in \operatorname{Spec}(\mathcal{A}),|\tau|>0\}
$$

and

$$
\alpha=\sup \left\{\beta \geq 0 ; \exists B>0 \forall u \in \mathcal{H}, \forall t \geq 0, E(u, t) \leq B e^{-\beta t} E(u, 0)\right\} .
$$

Here the space $\mathcal{H}$ of the Cauchy data is identified with solutions of (1.1). It was proved in [16] that

$$
\alpha=2 \min (D, A(\infty)) .
$$

In particular, it follows from (1.9) that if there exists a geodesic not intersecting the support of $a$, then $\alpha=A(\infty)=0$. In [16], an explicit example of a surface of revolution was given, where there exists a closed geodesic not meeting $\operatorname{supp}(a)$, while $D>0$, so that the eigenfrequencies are bounded away from the real axis. In the example the geodesic in question is hyperbolic. Our next result shows that if there exists a closed geodesic away from the damping region, which is elliptic, then we have a sequence of eigenfrequencies approaching rapidly the real axis.

The precise assumptions on the geodesic will be formulated in terms of the associated linearized Poincaré map, and we shall pause here to recall this notion. Given a simple closed $H_{p}$-trajectory $\gamma:[0, T] \rightarrow p^{-1}(1)$ (satisfying $\gamma(0)=\gamma(T)$ ), the linearized Poincaré map $P_{\gamma}$ is defined as follows: let $H \subset p^{-1}(1)$ be a smooth hypersurface intersecting $\gamma$ transversally at $\gamma(0)$. Then $P_{\gamma}$ is the differential at $\gamma(0)$ of the smooth locally defined Poincare map: $H \rightarrow H$, obtained by following the flow of $H_{p}$ once around $\gamma$. Up to a symplectic conjugation, the mapping $P_{\gamma}$ can be viewed as a linear 
symplectic transformation on $\mathbf{R}^{2(n-1)}$, and we say that $\gamma$ is elliptic and nondegenerate if the spectrum of $P_{\gamma}$ lies on the unit circle and 1 is not an eigenvalue.

Given a nondegenerate elliptic closed $H_{p}$-trajectory $\gamma$, with the eigenvalues of the corresponding $P_{\gamma}$ of the form $e^{ \pm i \alpha_{j}}, j=1, \ldots n-1$, and $0<\alpha_{j} \leq \pi$, we say that $P_{\gamma}$ is $N$-elementary if

$$
\sum_{j=1}^{n-1} k_{j} \alpha_{j} \neq 0 \quad \bmod 2 \pi \mathbf{Z}
$$

for each $k=\left(k_{1}, \ldots k_{n-1}\right) \in \mathbf{Z}^{n-1}$ with $0<\sum_{j=1}^{n-1}\left|k_{j}\right| \leq N$.

THEOREM 1.3. Suppose that there exists a closed primitive $H_{p}$-orbit $\gamma:[0, T] \rightarrow$ $p^{-1}(1)$, which is nondegenerate elliptic, and such that the associated mapping $P_{\gamma}$ is $N$-elementary for some $N \geq 4$. Assume that $\pi(\gamma([0, T])) \cap \operatorname{supp}(a)=\emptyset$. Then there exists a sequence $\left(\tau_{j}\right), j=1,2 \ldots$, of eigenfrequencies such that $\left|\operatorname{Re} \tau_{j}\right| \rightarrow \infty$, and $0<\operatorname{Im} \tau_{j} \leq \mathcal{O}_{N}(1)\left|\operatorname{Re} \tau_{j}\right|^{-N}, j \rightarrow \infty$, for any $N$.

REMARK. This theorem can be viewed as an analogue of the results of [24], [25], and $[23]$ in the theory of resonances.

Our last theorem is concerned with an expansion of the propagator $U(t)$ on a Zoll manifold, i.e., a manifold all of whose geodesics are closed. We refer to [3] for the background and motivation for the study of such manifolds. In this case, the set of the eigenfrequencies displays a cluster structure, and the expansion holds in terms of the disjoint clusters in the entire spectral band.

TheOREM 1.4. Let $M$ be a Zoll manifold, i.e. assume that $\exp \left(\pi H_{p}\right)(x, \xi)=$ $(x, \xi),(x, \xi) \in p^{-1}(1)$. Then

1. There exists $C>0$ such that all eigenfrequencies $\tau$, with $\operatorname{Re} \tau>0$, except for finitely many, are contained in the union of the rectangles

$$
I_{k}=\left[k+\frac{\alpha}{4}-\frac{C}{k}, k+\frac{\alpha}{4}+\frac{C}{k}\right]+i[0, \mathcal{O}(1)], \quad k=1,2, \ldots,
$$

where $\alpha \in \mathbf{Z}$ is the Maslov index of the closed $H_{p}$-trajectory

$$
\left\{\exp \left(t H_{p}\right)(x, \xi) ;(x, \xi) \in p^{-1}(1), t \in[0, \pi]\right\} .
$$

2. We have

$$
U(t)=\sum_{k=1}^{\infty}\left(\sum_{\tau \in \operatorname{Spec}(\mathcal{A}), \tau \in I_{k}} e^{i t \tau} p_{\tau, k}(t)+\sum_{\tau \in \operatorname{Spec}(\mathcal{A}),-\bar{\tau} \in I_{k}} e^{i t \tau} p_{\tau, k}(t)\right), t>0
$$

Here $p_{\tau, k}$ is a polynomial in $t$ with values in $\mathcal{L}\left(L^{2}, H^{s}\right)$ for any $s \in \mathbf{R}$. The outer sum in (1.11) converges absolutely in $\mathcal{L}\left(H^{\theta}, L^{2}\right)$ for every $\theta>0$ and for each fixed $t>0$.

REMARK. The first part of Theorem 1.4 has been established in [12]. This is a well-known and in general optimal result in the self-adjoint case, $a \equiv 0$ - see Chapter 29 of $[15]$. 
REMARK. The rectangles $I_{k}$ in (1.10) become disjoint only for $k$ large enough, $k \geq k_{0} \gg 1$, and the expansion in (1.11) should be understood in the following sense: If we set

$$
U_{k}(t)=\sum_{\tau \in \operatorname{Spec}(\mathcal{A}), \tau \in I_{k}} e^{i t \tau} p_{\tau, k}(t)+\sum_{\tau \in \operatorname{Spec}(\mathcal{A}),-\bar{\tau} \in I_{k}} e^{i t \tau} p_{\tau, k}(t), \quad k \geq k_{0},
$$

then the series

$$
\sum_{k=k_{0}}^{\infty} U_{k}(t)
$$

converges absolutely in $\mathcal{L}\left(H^{\theta}, L^{2}\right)$, for any $\theta>0$ and each $t>0$, and we have

$$
U(t)=\sum_{\tau \in \operatorname{Spec}(\mathcal{A}),|\operatorname{Re} \tau|<k_{0}+\alpha / 4} e^{i t \tau} p_{\tau}(t)+\sum_{k \geq k_{0}} U_{k}(t) \quad \text { in } \mathcal{L}\left(H^{\theta}, L^{2}\right) .
$$

The expansion result of Theorem 1.4 is valid only in a very special case of Zoll manifolds, and many open problems concerning eigenfunction expansions for the damped wave propagator remain. One might suspect that for manifolds with more general geometry and the location of the damping region, weaker expansions still may hold. In particular, abandoning convergence and following [5], one may look for timedependent expansions, where, rather than attempting to "cut" through a dense cloud of eigenfrequencies close to the real axis, one considers suitable sums of finitely many terms, with the number of terms depending on time. In the present work, we shall not try to attack this problem and would merely like to mention that it seems that as compared with [5], some additional features are likely to be present in our case, due to the fact that there is no underlying selfadjoint operator.

The plan of the paper is as follows. After some preliminaries in Section 2, Theorem 1.2 is proved in Section 3. The proof relies on a simple conjugation by pseudodifferential operators, microlocally in the region of the phase space, where we lack ellipticity. We also adapt the classical argument of Morawetz [18] to our situation, in order to obtain Theorem 1.2 in the case of the $L^{2}$ initial data. In Section 4 we study the case when the geodesic flow is periodic. Here we recall the methods of [12], and show how they can be used to prove the second part of Theorem 1.4. Section 5 is devoted to the proof of Theorem 1.3. The proof depends on an a priori semiclassical resolvent estimate, and here we follow the idea of [17] and [22] of creating gaps in the spectrum of the unperturbed operator by means of an auxiliary finite rank perturbation. At this stage we also use the results and methods of [6], [25], and [23].

The results of this paper have been announced in [13].

Acknowledgements. I am very grateful to Maciej Zworski for numerous helpful discussions on the subject of the present paper. I would also like to thank Nicolas Burq for pointing out the argument of Morawetz, a modification of which is used in Section 3. The support of the Swedish Foundation for International Cooperation in Research and Higher Education (STINT), of the MSRI postdoctoral fellowship, as well as a partial support of the National Science Foundation under grant DMS-0304970, is gratefully acknowledged.

2. Preliminaries. In the beginning of this section we shall recall, for future reference, some resolvent estimates for $\mathcal{A}$. In doing so we shall write $D=\{\tau \in$ $\left.\mathbf{C} ; \operatorname{Im} \tau \in\left[0,2\|a\|_{\infty}\right]\right\}$, and we recall that $\operatorname{Spec}(\mathcal{A}) \subset D$. 
Proposition 2.1. There exists a constant $C>0$ such that

$$
\left\|(\tau-\mathcal{A})^{-1}\right\|_{\mathcal{L}(\mathcal{H}, \mathcal{H})} \leq \frac{C}{\operatorname{dist}(\tau, D)}, \quad \tau \notin D
$$

Proof. Let us consider the following closed codimension one subspace of $\mathcal{H}=$ $H^{1} \times L^{2}$,

$$
\mathcal{H}_{0}=\left\{\left(u_{0}, u_{1}\right) \in \mathcal{H} ; \int 2 i a(x) u_{0}(x) d x-\int u_{1}(x) d x=0\right\}
$$

Since $a \geq 0$ is not identically zero, it is true that the inner product on $\mathcal{H}_{0}$,

$$
\left\langle\left(u_{0}, u_{1}\right),\left(v_{0}, v_{1}\right)\right\rangle_{E}:=\int \nabla u_{0} \overline{\nabla v_{0}} d x+\int u_{1} \overline{v_{1}} d x
$$

defines a norm $\|\cdot\|_{E}$ there which is equivalent to the natural norm on $\mathcal{H},\|\cdot\|_{\mathcal{H}}$. When $U=\left(u_{0}, u_{1}\right) \in D(\mathcal{A})$, it is also true that $\mathcal{A} U=\left(u_{1},-\Delta u_{0}+2 i a u_{1}\right)$ is in $\mathcal{H}_{0}$, and a simple computation shows that $\mathcal{H}_{0}$ contains $\bigoplus_{0 \neq \tau \in \operatorname{Spec}(\mathcal{A})} E_{\tau}$ as a (dense) subspace. When $U \in D(\mathcal{A}) \cap \mathcal{H}_{0}$, we have

$$
0 \leq \operatorname{Im}\langle\mathcal{A} U, U\rangle_{E} \leq 2\|a\|_{\infty}\|U\|_{E}^{2}
$$

so that when $\operatorname{Im} \tau<0$, we get $\operatorname{Im}\langle(\mathcal{A}-\tau) U, U\rangle_{E} \geq|\operatorname{Im} \tau|\langle U, U\rangle_{E}$. It follows that there exists $C>0$ such that for any $U \in \mathcal{H}_{0}$,

$$
\left\|(\tau-\mathcal{A})^{-1} U\right\|_{\mathcal{H}} \leq \frac{C}{|\operatorname{Im} \tau|}\|U\|_{\mathcal{H}}, \quad \operatorname{Im} \tau<0 .
$$

Now it is easily seen that the space $E_{0}$ is of dimension one and is generated by $(1,0)$. We have $\mathcal{H}=\mathcal{H}_{0} \oplus E_{0}$, also in the topological sense, and since

$$
\left\|(\tau-\mathcal{A})^{-1} V\right\|_{\mathcal{H}} \leq \frac{1}{|\operatorname{Im} \tau|}\|V\|_{\mathcal{H}}, \quad \operatorname{Im} \tau<0, \quad V \in E_{0},
$$

we conclude that (2.1) follows from (2.2) and (2.3), in the case when $\operatorname{Im} \tau<0$. The proof is complete, for in the case when $\operatorname{Im} \tau>2\|a\|_{\infty}$, we argue in a similar way.

REMARK. A straightforward computation shows that

$$
(\tau-\mathcal{A})^{-1}=\left(\begin{array}{cc}
R(\tau)(2 i a-\tau) & -R(\tau) \\
R(\tau)\left(2 i a \tau-\tau^{2}\right)-1 & -R(\tau) \tau
\end{array}\right)
$$

where

$$
R(\tau)=P(\tau)^{-1}, \quad P(\tau)=-\Delta+2 i a \tau-\tau^{2} .
$$

Combining (2.4) with Proposition 2.1 we get

$$
\|R(\tau)\|_{\mathcal{L}\left(L^{2}, L^{2}\right)} \leq \frac{C}{|\tau||\operatorname{Im} \tau|}, \quad \operatorname{Im} \tau<0
$$

and

$$
\|R(\tau)\|_{\mathcal{L}\left(L^{2}, L^{2}\right)} \leq \frac{C}{|\tau|\left(\operatorname{Im} \tau-2\|a\|_{\infty}\right)}, \quad \operatorname{Im} \tau>2\|a\|_{\infty}
$$


It follows from the proof of Proposition 2.1 that

$$
\left\|(\tau-\mathcal{A})^{-n}\right\|_{\mathcal{L}(\mathcal{H}, \mathcal{H})} \leq \frac{C}{\operatorname{dist}(\tau, D)^{n}}, \quad \tau \notin D, n=1,2, \ldots,
$$

and an application of the Hille-Yosida theorem shows that $\mathcal{A}$ generates a uniformly bounded strongly continuous semigroup $\mathcal{U}(t)=e^{i t \mathcal{A}}, t \geq 0$, in $\mathcal{H}$, so that in particular $U(t) \in C\left(\overline{\mathbf{R}_{+}}, \mathcal{L}\left(L^{2}, H^{1}\right)\right) \cap C^{1}\left(\overline{\mathbf{R}_{+}}, \mathcal{L}\left(L^{2}, L^{2}\right)\right)$, in the strong sense. See [9]. We have the following relation between $\mathcal{U}(t)$ and the resolvent of $\mathcal{A}$, valid when $x \in D\left(\mathcal{A}^{2}\right)$,

$$
e^{i t \mathcal{A}} x=x+\frac{1}{2 \pi i} \int_{-\infty-i \alpha}^{\infty-i \alpha} e^{i t \tau}(\tau-\mathcal{A})^{-1} \mathcal{A} x \frac{d \tau}{\tau}, \quad t>0, \alpha>0
$$

The integral in (2.8) converges absolutely in $\mathcal{H}$. If we choose $x=(0, f), f \in H^{2}$, then (2.4) shows that the first component of $(\tau-\mathcal{A})^{-1} \mathcal{A} x$ is equal to $-\tau R(\tau) f$. Thus, with the absolute convergence in $L^{2}$ we have,

$$
U(t) f=\frac{i}{2 \pi} \int_{-\infty-i \alpha}^{\infty-i \alpha} e^{i t \tau} R(\tau) f d \tau, \quad \alpha>0, \quad f \in H^{2}(M) .
$$

Notice that when $f \in L^{2}(M),(2.9)$ still holds, with the integral in (2.9) converging in $L^{2}\left(\operatorname{Im} \tau=-\alpha ; L^{2}(M)\right)$.

When establishing our results, it will be convenient to work in the semiclassical setting. We shall therefore perform a semiclassical reduction, following [22] and [12]. When considering the eigenvalue problem

$$
\left(-\Delta+2 i a(x) \tau-\tau^{2}\right) u(x)=0,
$$

with $\operatorname{Im} \tau=\mathcal{O}(1)$ and $\operatorname{Re} \tau \gg 1$, we write $\tau=\sqrt{z} / h$, where $0<h \ll 1$, and $z$ belongs to the fixed domain $[\alpha, \beta]+i[-\gamma, \gamma], 0<\alpha<1<\beta<\infty, \gamma>0$. We are then led to study the $h$-differential operator

$$
\mathcal{P}=P+i h Q(z)
$$

where $P=-h^{2} \Delta$ and $Q(z)=2 a(x) \sqrt{z}$. We notice that $P$ is essentially selfadjoint on $C^{\infty}(M)$, with the domain of the selfadjoint realization being $H^{2}(M)$, and $Q(z)$ is bounded and selfadjoint for $z$ real positive.

In the main part of the paper, we shall make use of some elementary semiclassical pseudodifferential calculus, and we digress here to recall some relevant notation. Let $S^{m}\left(T^{*} M\right)=S_{1,0}^{m}\left(T^{*} M\right)$ be the space of functions $a(x, \xi ; h)$ on $T^{*} M \times\left(0, h_{0}\right], h_{0}>0$, which are smooth in $(x, \xi)$ and such that

$$
\partial_{x}^{\alpha} \partial_{\xi}^{\beta} a(x, \xi ; h)=\mathcal{O}_{\alpha, \beta}(1)(1+|\xi|)^{m-|\beta|}, \quad(x, \xi) \in T^{*} M, h \in\left(0, h_{0}\right] .
$$

When $a$ depends on some additional parameters, we require the estimates (2.11) to hold uniformly with respect to these parameters. The formula for the classical $h$ quantization,

$$
a\left(x, h D_{x} ; h\right) u(x)=\frac{1}{(2 \pi h)^{n}} \iint e^{i \frac{\langle x-y, \xi\rangle}{h}} a(x, \xi ; h) u(y) d y d \xi,
$$

then defines a class of $h$-pseudodiffential operators on $M$, which will be denoted by $\mathrm{Op}_{h}\left(S^{m}\right)$. 
For $h$-dependent symbols, we write $a \in S_{\mathrm{cl}}^{m}$ if there exists $a_{0} \in S^{m}$, independent of $h$, such that $a-a_{0} \in h S^{m-1}$, and we say that $a_{0}$ is the principal symbol of $a$ and of the corresponding operator. When $A \in \mathrm{Op}_{h}\left(S^{m}\right)$ has a principal symbol $a_{0} \in S^{m}$, we write $A=\mathrm{Op}_{h}\left(a_{0}\right)$ and say that $A$ is classical.

We shall finally recall the notion of the (semiclassical) wave front set, $W F_{h}(A)$, of an operator $A \in \mathrm{Op}_{h}\left(S^{m}\right)$. When $\rho \in T^{*} M$, we say that $\rho \notin W F_{h}(A)$, if the full symbol of $A$, for some choice of local coordinates near the projection of $\rho$, is of class $S^{-\infty, m}:=\cap_{k} h^{k} S^{m}$ near $\rho$. It follows from the definition that $W F_{h}(A)$ is a closed subset of $T^{*} M, W F_{h}(A B) \subset W F_{h}(A) \cap W F_{h}(B)$, and $W F_{h}(A)=\emptyset \Rightarrow h^{-k} A \in$ $\mathrm{Op}_{h}\left(S^{m}\right)$, for any $k$. In this paper, we shall work in a purely semiclassical setting, and consequently, only compact subsets of $T^{*} M$ will be important. The definition of $W F_{h}$ given above will therefore be sufficient for our purposes.

3. Proof of Theorem 1.2. The main step in the proof of Theorem 1.2 consists of establishing the following result.

Proposition 3.1. Assume that the Rauch-Taylor condition holds. Then for every $\varepsilon \in(0, A(\infty))$ there exists $C=C(\varepsilon)>0$ such that if $\operatorname{Im} \tau \in[-1, A(\infty)-\varepsilon]$ and $|\operatorname{Re} \tau| \geq C$ then

$$
\|R(\tau)\|_{\mathcal{L}\left(L^{2}, L^{2}\right)} \leq \frac{C}{1+|\tau|}
$$

Proof. Performing the semiclassical reduction of Section 2, we can reformulate the statement of the proposition in the following form: for every $\varepsilon \in(0, A(\infty))$ there exists $C>0$ and $h_{0}>0$ such that for all $h \in\left(0, h_{0}\right]$ and all

$$
z \in \Omega(\varepsilon):=\{\operatorname{Re} z \in[\alpha, \beta],-2 \sqrt{\operatorname{Re} z} \leq \operatorname{Im} z / h \leq 2 \sqrt{\operatorname{Re} z}(A(\infty)-\varepsilon)\},
$$

it is true that

$$
\left\|(\mathcal{P}-z)^{-1}\right\|_{\mathcal{L}\left(L^{2}, L^{2}\right)} \leq \frac{C}{h}
$$

Here $\mathcal{P}$ has been defined in (2.10). The proof of (3.3), sketched in [13] was a contradiction argument, and it relied upon a propagation estimate for a suitable semiclassical measure. Here we shall give a different proof, which has the merit of being more direct. In doing so, we shall use some arguments of [22] and [12].

In what follows we shall assume, as we may, that $\operatorname{Im} z=\mathcal{O}(h)$. Following the argument of Section 2 of [22], we conjugate $\mathcal{P}=P+i h Q(z)$ by an elliptic selfadjoint $h$-pseudodifferential operator $A=\mathrm{Op}_{h}\left(e^{g}\right)$, where $g \in S^{-1}\left(T^{*} M\right)$ is to be chosen. We have

$$
A^{-1}(P+i h Q(z)) A=P+i h \mathrm{Op}_{h}\left(q(\operatorname{Re} z)-H_{p} g\right)+h^{2} R(z) .
$$

Here $q(z)=2 a \sqrt{z}$ and $R(z) \in \mathrm{Op}_{h}\left(S^{0}\right)$. Let us choose some $\delta>0$, sufficiently small but fixed. Continuing to follow the argument of [22], we see that we can choose $g=g(\operatorname{Re} z) \in S^{-1}$ such that

$$
q(\operatorname{Re} z)-H_{p} g=\langle q(\operatorname{Re} z)\rangle_{T} \quad \text { on } p^{-1}([\alpha-\delta, \beta+\delta]) .
$$

Here the trajectory average $\langle q(\operatorname{Re} z)\rangle_{T}$ is given by

$$
\langle q(\operatorname{Re} z)\rangle_{T}=\frac{1}{T} \int_{0}^{T} q(\operatorname{Re} z) \circ \exp \left(t H_{p}\right) d t
$$


and $T>0$ is to be chosen later, large enough but fixed. We then consider the right hand side of (3.4),

$$
\widehat{\mathcal{P}}=\widehat{\mathcal{P}}_{T}=P+i h \mathrm{Op}_{h}\left(\widehat{q}_{T}\right)+h^{2} R_{T}(z),
$$

where $\widehat{q}_{T} \in S^{0}$ is such that

$$
\widehat{q}_{T}=\langle q(\operatorname{Re} z)\rangle_{T} \quad \text { on } p^{-1}([\alpha-\delta, \beta+\delta]) .
$$

We now claim that

$$
\lim _{T \rightarrow \infty} \inf _{p^{-1}(E)}\langle q(\operatorname{Re} z)\rangle_{T}=2 \sqrt{\operatorname{Re} z} A(\infty),
$$

locally uniformly in $E>0$. Indeed, we observe that the homogeneity property of the $H_{p}$-flow implies that

$$
\inf _{p^{-1}(E)}\langle a\rangle_{T}=\inf _{p^{-1}(1)}\langle a\rangle_{\sqrt{E} T},
$$

and choosing $T$ large enough but fixed, depending on $\varepsilon>0$, we can therefore arrange that for $\widehat{q}_{T}$ in (3.5), we have

$$
\widehat{q}_{T} \geq 2 \sqrt{\operatorname{Re} z}(A(\infty)-\varepsilon / 2) \quad \text { on } p^{-1}([\alpha-\delta, \beta+\delta]) .
$$

Take now a cutoff function $0 \leq \chi \in C_{0}^{\infty}\left(p^{-1}((\alpha-\delta, \beta+\delta))\right)$, such that $\chi=1$ near $p^{-1}([\alpha, \beta])$. An application of the Gårding inequality allows us to conclude that for $z \in \Omega(\varepsilon)$,

$$
(\operatorname{Im}(\widehat{\mathcal{P}}-z) \chi u \mid \chi u) \geq \eta h\|\chi u\|^{2}-\mathcal{O}\left(h^{\infty}\right)\|u\|^{2},
$$

where $\eta=\eta(\varepsilon)>0$ is fixed and $h>0$ is small enough, depending on $\varepsilon$. Here we let $\chi$ also stand for the corresponding quantization, and the inner product and the norm in (3.6) are taken in $L^{2}(M)$. On the other hand, we have

$$
(\operatorname{Im}(\widehat{\mathcal{P}}-z) \chi u \mid \chi u)=\operatorname{Im}((\chi(\widehat{\mathcal{P}}-z) u \mid \chi u)+([\widehat{\mathcal{P}}, \chi] u \mid \chi u)),
$$

and the absolute value of this expression does not exceed

$$
\mathcal{O}(1)\|(\widehat{\mathcal{P}}-z) u\|\|\chi u\|+\mathcal{O}(1)\|[\widehat{\mathcal{P}}, \chi] u\|\|\chi u\| .
$$

Let now $\chi_{1} \in C_{0}^{\infty}\left(p^{-1}((\alpha-\delta, \beta+\delta))\right.$ be such that $\chi_{1}=1$ near $p^{-1}([-\alpha, \beta])$ and the support of $\chi_{1}$ is contained in the interior of the set where $\chi=1$. Then

$$
\|[\widehat{\mathcal{P}}, \chi] u\| \leq\left\|[\widehat{\mathcal{P}}, \chi]\left(1-\chi_{1}\right) u\right\|+\mathcal{O}\left(h^{\infty}\right)\|u\| \leq \mathcal{O}(h)\left\|\left(1-\chi_{1}\right) u\right\|+\mathcal{O}\left(h^{\infty}\right)\|u\| .
$$

Now the operator $\widehat{\mathcal{P}}-z$ is elliptic away from $p^{-1}([\alpha, \beta])$, and the semiclassical elliptic regularity shows that

$$
\left\|\left(1-\chi_{1}\right) u\right\| \leq \mathcal{O}(1)\|(\widehat{\mathcal{P}}-z) u\|+\mathcal{O}\left(h^{\infty}\right)\|u\| .
$$

We get

$$
\|[\widehat{\mathcal{P}}, \chi] u\| \leq \mathcal{O}(h)\|(\widehat{\mathcal{P}}-z) u\|+\mathcal{O}\left(h^{\infty}\right)\|u\| .
$$


Combining (3.6) together with (3.7) and (3.9), we infer that

$$
\begin{aligned}
\|\chi u\|^{2} & \leq \frac{\mathcal{O}(1)}{h}\|(\widehat{\mathcal{P}}-z) u\|\|\chi u\|+\mathcal{O}\left(h^{\infty}\right)\|u\|^{2} \\
& \leq \frac{\mathcal{O}(1)}{h^{2}}\|(\widehat{\mathcal{P}}-z) u\|^{2}+\frac{1}{2}\|\chi u\|^{2}+\mathcal{O}\left(h^{\infty}\right)\|u\|^{2},
\end{aligned}
$$

and therefore

$$
\|\chi u\| \leq \frac{\mathcal{O}(1)}{h}\|(\widehat{\mathcal{P}}-z) u\|+\mathcal{O}\left(h^{\infty}\right)\|u\| .
$$

Combining this with (3.8), with $\chi$ in place of $\chi_{1}$, we get

$$
\|u\| \leq \frac{\mathcal{O}(1)}{h}\|(\widehat{\mathcal{P}}-z) u\|, \quad u \in H^{2}(M), \quad z \in \Omega(\varepsilon) .
$$

This completes the proof if we take into account the uniform bounds

$$
A, A^{-1}=\mathcal{O}_{s}(1): H^{s} \rightarrow H^{s}, \quad s \in \mathbf{R},
$$

where the $H^{s}$-spaces have been equipped with their natural $h$-dependent norms.

It is now easy to obtain an expansion of $U(t)$ in $\mathcal{L}\left(H^{\theta}, L^{2}\right), \theta>0$, as $t \rightarrow \infty$. Indeed, if $f \in H^{2}$ then

$$
R(\tau)\left(-\Delta+2 i a \tau-\tau^{2}\right) f=f
$$

which together with Proposition 3.1 implies that for $|\operatorname{Re} \tau| \geq C$ and $\operatorname{Im} \tau \in$ $[-1, A(\infty)-\varepsilon]$, we have

$$
\|R(\tau)\|_{\mathcal{L}\left(H^{2}, L^{2}\right)} \leq \frac{\mathcal{O}(1)}{(1+|\tau|)^{2}} .
$$

Interpolating between (3.1) and (3.10), we get

$$
\|R(\tau)\|_{\mathcal{L}\left(H^{\theta}, L^{2}\right)} \leq \frac{\mathcal{O}(1)}{(1+|\tau|)^{1+\theta / 2}}, \quad 0 \leq \theta \leq 2,
$$

so that for $\theta>0$,

$$
\int_{-\infty-i \alpha}^{\infty-i \alpha} e^{i t \tau} R(\tau) d \tau \in C\left(\overline{\mathbf{R}}_{+}, \mathcal{L}\left(H^{\theta}, L^{2}\right)\right), \quad \alpha>0 .
$$

The expansion for $U(t)$ stated in Theorem 1.2, with an error estimate (1.8) in $\mathcal{L}\left(H^{\theta}, L^{2}\right)$, is then obtained by deforming the contour of integration in the right hand side of (2.9), exactly as in the proof of Theorem 1 of [26].

We now come to prove Theorem 1.2 in the Banach space $\mathcal{L}\left(L^{2}, L^{2}\right)$. In doing so, we shall make use of an argument which comes essentially from [18], there given in the context of the wave equation in the exterior of a nontrapping obstacle. See also [4]. We shall transform the initial value problem (1.1) into a non-homogeneous problem.

Let $\varepsilon \in(0, A(\infty))$ be such that there are no eigenfrequencies $\tau$ with the imaginary part $\operatorname{Im} \tau=A(\infty)-\varepsilon$, and let $\tau_{1}, \ldots \tau_{N}$ be the eigenfrequencies with $\operatorname{Im} \tau_{j}<A(\infty)-\varepsilon$. We introduce the spectral projector onto $\bigoplus_{j=1}^{N} E_{\tau_{j}}$,

$$
\Pi=\frac{1}{2 \pi i} \int_{\gamma}(\tau-\mathcal{A})^{-1} d \tau
$$


where $\gamma$ consists of the union of small positively oriented circles centered at the $\tau_{j}$ 's. When $x \in \mathcal{H}$, it is true that

$$
(\tau-\mathcal{A})^{-1}(I-\Pi) x
$$

is a holomorphic function in a neigbourhood of the set where $\operatorname{Im} \tau \leq A(\infty)-\varepsilon$, with values in $\mathcal{H}$. We shall now derive weighted decay estimates for $e^{i t \overline{\mathcal{A}}}(I-\Pi) x$. When doing so, we notice first that Proposition 3.1 together with an interpolation argument shows that

$$
R(\tau)=\mathcal{O}(1): L^{2} \rightarrow H^{1}, \quad|\operatorname{Re} \tau| \geq \mathcal{O}(1)
$$

when $\operatorname{Im} \tau \leq A(\infty)-\varepsilon$. Let $\psi \in C^{\infty}(\mathbf{R} ; \mathbf{R})$ be an increasing function such that $\psi(t)=0, t \leq 0$, and $\psi(t)=1, t \geq 1$. We consider the function $\psi(t) u(t, x)$ where $u(t, x)$ solves the evolution problem (1.1) with the initial data $\left(u_{0}, u_{1}\right)=(I-\Pi) x$, when $x=(0, f), f \in L^{2}$. Then

$$
\left(-D_{t}^{2}-\Delta+2 i a(x) D_{t}\right) \psi(t) u(t, x)=g(t, x)
$$

where $g \in C\left(\mathbf{R} ; L^{2}\right)$ is given by

$$
g(t, x)=\psi^{\prime}(t)\left(2 a(x) u(t, x)+i D_{t} u(t, x)\right)+i D_{t}\left(\psi^{\prime}(t) u(t, x)\right) .
$$

The support of $g(\cdot, x)$ is contained in $[0,1]$ so that $g \in L^{2}\left(\mathbf{R} ; L^{2}\right)$, and it follows from the Hille-Yosida theorem that

$$
\|g\|_{L^{2}\left(\mathbf{R} ; L^{2}\right)} \leq \mathcal{O}(1)\|f\|_{L^{2}} .
$$

Taking the Fourier transform in $t$ of $(3.12), \mathcal{F}_{t \rightarrow \tau}$, we get

$$
P(\tau) \mathcal{F}_{t \rightarrow \tau} \psi(t) u(t, x)=\mathcal{F}_{t \rightarrow \tau} g(t, x), \quad \mathcal{F}_{t \rightarrow \tau} g(t, x) \in L^{2}\left(\mathbf{R} ; L^{2}\right) .
$$

Now an application of (2.4) shows that $R(\tau) \mathcal{F}_{t \rightarrow \tau} g(t, x)=P(\tau)^{-1} \mathcal{F}_{t \rightarrow \tau} g(t, x)$ is the first component of

$$
\frac{1}{i}(\tau-\mathcal{A})^{-1} \mathcal{F}_{t \rightarrow \tau} \psi^{\prime}(t)\left(\begin{array}{c}
u(t, x) \\
D_{t} u(t, x)
\end{array}\right)
$$

so that it depends holomorphically on $\tau$ in the set where $\operatorname{Im} \tau \leq A(\infty)-\varepsilon$, and takes values in $H^{1}$.

An application of Parseval's formula to (3.14) together with (3.11) and (3.13) gives that

$$
\begin{aligned}
& \left\|e^{(A(\infty)-\varepsilon) t} \psi(t) u(t)\right\|_{L^{2}\left(\mathbf{R} ; H^{1}\right)} \\
= & \left\|\left.R(\tau) \mathcal{F}_{t \rightarrow \tau} g(t, \cdot)\right|_{\tau=+i(A(\infty)-\varepsilon)}\right\|_{L^{2}\left(\mathbf{R} ; H^{1}\right)} \\
\leq & \mathcal{O}(1)\left\|\left.\mathcal{F}_{t \rightarrow \tau} g(t, \cdot)\right|_{\tau=++i(A(\infty)-\varepsilon)}\right\|_{L^{2}\left(\mathbf{R} ; L^{2}\right)} \leq \mathcal{O}(1)\|f\|_{L^{2}}
\end{aligned}
$$

This gives an exponential decay estimate in the integrated form. When deriving estimates that are pointwise in time, we consider the non-homogeneous equation satisfied by $\psi_{T}(t) u(t, x)$, where $\psi_{T}(t)=\psi(t-T), T>1$. We have

$$
\left(-D_{t}^{2}-\Delta+2 i a(x) D_{t}\right) \psi_{T}(t) u(t, x)=g_{T}(t, x),
$$


where $g_{T}(t, x)=\left(\psi_{T}^{\prime \prime}(t)+2 a(x) \psi_{T}^{\prime}(t)\right) u(t, x)+2 i \psi_{T}^{\prime}(t) D_{t} u(t, x)$. Standard hyperbolic estimates (see [15]) show that

$$
\|u(T+1, \cdot)\|_{L^{2}} \leq \mathcal{O}(1) \int_{T}^{T+1}\left\|g_{T}(t, \cdot)\right\|_{L^{2}} d t \leq \mathcal{O}(1)\left(\int_{T}^{T+1}\left\|g_{T}(t, \cdot)\right\|_{L^{2}}^{2} d t\right)^{1 / 2}
$$

and therefore

$$
\|u(T+1, \cdot)\|_{L^{2}}^{2} \leq \mathcal{O}(1)\left(\int_{T}^{T+1}\left(\|u(t, \cdot)\|_{L^{2}}^{2}+\left\|D_{t} u(t, \cdot)\right\|_{L^{2}}^{2}\right) d t\right) .
$$

Now it follows from (3.15) that

$$
\begin{aligned}
\int_{T}^{T+1}\|u(t, \cdot)\|_{L^{2}}^{2} d t & \leq e^{-2(A(\infty)-\varepsilon) T} \int_{0}^{\infty} e^{2(A(\infty)-\varepsilon) t} \psi^{2}(t)\|u(t, \cdot)\|_{L^{2}}^{2} d t \\
& \leq \mathcal{O}(1) e^{-2(A(\infty)-\varepsilon) T}\|f\|_{L^{2}}^{2},
\end{aligned}
$$

and we shall now verify that the corresponding estimate holds when $u(t, \cdot)$ is replaced by $D_{t} u(t, \cdot)$. In doing so, we observe that (3.14) gives

$$
P(\tau) \mathcal{F}_{t \rightarrow \tau}\left(D_{t}(\psi(t) u(t, x))\right)=\tau \mathcal{F}_{t \rightarrow \tau} g(t, x)
$$

and since

$$
\tau R(\tau)=\mathcal{O}(1): L^{2} \rightarrow L^{2}, \quad|\operatorname{Re} \tau| \geq \mathcal{O}(1), \operatorname{Im} \tau \in[-1, A(\infty)-\varepsilon],
$$

repeating the previous arguments we see that

$$
\int_{0}^{\infty} e^{2(A(\infty)-\varepsilon) t}\left\|D_{t}(\psi(t) u(t, \cdot))\right\|_{L^{2}}^{2} d t \leq \mathcal{O}(1)\|f\|_{L^{2}}^{2} .
$$

Since $\psi^{\prime}$ is supported in $[0,1]$, we get

$$
\int_{0}^{\infty} e^{2(A(\infty)-\varepsilon) t} \psi^{2}(t)\left\|D_{t} u(t, \cdot)\right\|_{L^{2}}^{2} d t \leq \mathcal{O}(1)\|f\|_{L^{2}}^{2}
$$

and therefore

$$
e^{2(A(\infty)-\varepsilon) T} \int_{T}^{T+1}\left\|D_{t} u(t, \cdot)\right\|_{L^{2}}^{2} d t \leq \mathcal{O}(1)\|f\|_{L^{2}}^{2} .
$$

Combining (3.16) with (3.17) and (3.18) we conclude that

$$
\|u(t, \cdot)\|_{L^{2}} \leq \mathcal{O}(1) e^{-(A(\infty)-\varepsilon) t}\|f\|_{L^{2}} .
$$

In order to derive an expansion of Theorem 1.2, we now only have to consider

$$
e^{i t \mathcal{A}} \Pi x=\frac{1}{2 \pi i} \int_{-\infty-i \alpha}^{\infty-i \alpha} e^{i t \tau}(\tau-\mathcal{A})^{-1} \Pi x d \tau, \quad \alpha>0, \quad x=(0, f),
$$

which is given by the sum of the residues of the integrand at the poles $\tau_{j}, j=1, \ldots N$. If $\tau_{0}$ is such a pole, then near $\tau_{0}$, we can write for some $M \in \mathbf{N}$, not exceeding the multiplicity of $\tau_{0}$,

$$
(\tau-\mathcal{A})^{-1} \equiv \frac{\Pi_{0}}{\tau-\tau_{0}}+\sum_{j=1}^{M} \frac{D_{j}}{\left(\tau-\tau_{0}\right)^{j+1}}
$$


modulo a function holomorphic in a neighborhood of $\tau_{0}$. Here $\Pi_{0}$ is the spectral projector onto $E_{\tau_{0}}$ and $D_{j}=\left(\mathcal{A}-\tau_{0}\right)^{j} \Pi_{0}$. The corresponding contribution to the sum of the residues is of the form $e^{i t \tau_{o}}$ times a polynomial of degree at most $M$ in $t$ with values in $\mathcal{L}\left(\mathcal{H}, D\left(\mathcal{A}^{\infty}\right)\right)$. In particular, when $\tau_{0}=0$, it is true that the corresponding generalized eigenspace $E_{0}$ is spanned by $(1,0)$ so that the pole at 0 is simple, and the spectral projector $\Pi_{0}$ is given by

$$
\Pi_{0}\left(\begin{array}{l}
u_{0} \\
u_{1}
\end{array}\right)=\left(\begin{array}{l}
c \\
0
\end{array}\right)
$$

where

$$
c=\frac{\int 2 i a(x) u_{0}(x) d x-\int u_{1}(x) d x}{\int 2 i a(x) d x} .
$$

Combining these remarks together with (3.19), we obtain the statement of Theorem 1.2 .

4. Proof of Theorem 1.4. In this section we shall work under the assumption that the geodesic flow on $M$ is periodic. To fix the ideas, we shall assume throughout that $\exp \left(\pi H_{p}\right)(x, \xi)=(x, \xi),(x, \xi) \in p^{-1}(1)$. Our purpose is to derive an expansion for the damped wave propagator $U(t)$ on $M$. In doing so, we shall make use of the methods of [12]. Let us recall that in [12] it was established that

$$
\operatorname{Spec}(\mathcal{A}) \cap\{\tau \in \mathbf{C} ; \operatorname{Re} \tau>0\} \subset \bigcup_{k=1}^{\infty} I_{k}
$$

where

$$
I_{k}=\left[k+\frac{\alpha}{4}-\frac{\mathcal{O}(1)}{k}, k+\frac{\alpha}{4}+\frac{\mathcal{O}(1)}{k}\right]+i[0, \mathcal{O}(1)], \quad \alpha \in \mathbf{Z}, \quad k=1,2, \ldots
$$

We shall now complement this result by deriving resolvent estimates in the gaps between the rectangles $I_{k}$.

Proposition 4.1. There exists $C>0$ such that when $\operatorname{Im} \tau \in\left[-1,2\|a\|_{L^{\infty}}+1\right]$ we have

$$
\left.\|R(\tau)\|_{\mathcal{L}\left(L^{2}, L^{2}\right)} \leq \frac{C}{1+|\tau|}, \quad|\operatorname{Re} \tau| \geq C, \quad \operatorname{dist}\left(|\operatorname{Re} \tau|, \cup_{k=1}^{\infty} J_{k}\right)\right) \geq \frac{1}{C}
$$

Here $J_{k}=\left[k+\frac{\alpha}{4}-\frac{C}{k}, k+\frac{\alpha}{4}+\frac{C}{k}\right]$.

Proof. Applying the semiclassical reduction of Section 2, we see that in order to prove (4.1) it suffices to establish the existence of $C>0$ and $h_{0}>0$ such that for all $h \in\left(0, h_{0}\right]$ we have that

$$
\left\|(\mathcal{P}-z)^{-1}\right\|_{\mathcal{L}\left(L^{2}, L^{2}\right)} \leq \frac{C}{h}
$$

when $\operatorname{Im} z=\mathcal{O}(h)$ and dist $\left(\operatorname{Re} z, \cup_{k=1}^{\infty} J_{k}(h)\right) \geq h / C$. Here the intervals

$$
J_{k}(h)=\left[h^{2}\left(k+\frac{\alpha}{4}\right)^{2}-\mathcal{O}\left(h^{2}\right), h^{2}\left(k+\frac{\alpha}{4}\right)^{2}+\mathcal{O}\left(h^{2}\right)\right], \quad k \sim \frac{1}{h},
$$


have width $\mathcal{O}\left(h^{2}\right)$ and are separated from each other by a distance which is $\sim h$. We also recall from [27] and [12] that

$$
\operatorname{Spec}(P) \cap[\alpha, \beta] \subset \cup_{k=1}^{\infty} J_{k}(h) .
$$

When proving the bound (4.2), we follow the suggestion of the remark given after Theorem 3.2 in [12], which in turn is based on the methods of Sections 2 and 3 of [22]. Thus, repeating the arguments of the remark in [12], we find that there exists an elliptic selfadjoint $h$-pseudodifferential operator $A=\mathrm{Op}_{h}\left(e^{g}\right)$, with $g=g(\operatorname{Re} z) \in$ $S^{-1}\left(T^{*} M\right)$, such that

$$
A^{-1}(P+i h Q(z)) A=P+i h \widehat{Q}+h^{2} R(z), \quad R(z) \in \mathrm{Op}_{h}\left(S^{0}\right),
$$

where $\widehat{Q}=\mathrm{Op}_{h}(\hat{q})$ is selfadjoint, and $S^{0} \ni \hat{q}$ is such that

$$
\hat{q}=\langle q\rangle_{T}=\frac{1}{T} \int_{0}^{T} q \circ \exp \left(t H_{p}\right) d t \quad \text { on } p^{-1}(\operatorname{Re} z) .
$$

Here $q=q(\operatorname{Re} z)=2 a \sqrt{\operatorname{Re} z}$ and $T=T(\operatorname{Re} z)$ is the common period of the closed $H_{p}$-trajectories in $p^{-1}(\operatorname{Re} z)$. As in the proof of Proposition 3.1, we choose $g$ satisfying $H_{p} g=q-\langle q\rangle_{T}$ on $p^{-1}(\operatorname{Re} z)$, and we may take for example,

$$
g=\frac{1}{T} \int_{0}^{T} t q\left(\exp \left(t H_{p}\right)\right) d t \quad \text { on } \quad p^{-1}(\operatorname{Re} z)
$$

Since

$$
A, A^{-1}=\mathcal{O}(1): L^{2} \rightarrow L^{2}
$$

it suffices to show that the bound (4.2) holds for the resolvent of

$$
\widehat{\mathcal{P}}=P+i h \widehat{Q}+h^{2} R(z) .
$$

Now $H_{p} \hat{q}=0$ on $p^{-1}(\operatorname{Re} z)$, and we have

$$
H_{p} \hat{q}=k(p-\operatorname{Re} z),
$$

with $k \in S^{-1}$. If we let $K \in \mathrm{Op}_{h}\left(S^{-1}\right)$ have $k$ as a principal symbol, then

$$
i h[P, \widehat{Q}]=h^{2} K(P-\operatorname{Re} z)+h^{3} S,
$$

where $S \in \mathrm{Op}_{h}\left(S^{0}\right)$. Now, if $A$ and $B$ are bounded selfadjoint operators, we have

$$
\|(A+i B) u\|^{2}=\|A u\|^{2}+\|B u\|^{2}+i([A, B] u, u)
$$

and using this identity together with (4.4) we get,

$$
\begin{aligned}
& 2\|(\widehat{\mathcal{P}}-z) u\|^{2} \geq\|(P+i h \widehat{Q}-z) u\|^{2}-\mathcal{O}\left(h^{4}\right)\|u\|^{2} \\
\geq & \|(P-\operatorname{Re} z) u\|^{2}+i h([P, \widehat{Q}] u, u)-\mathcal{O}\left(h^{4}\right)\|u\|^{2} \\
\geq & \|(P-\operatorname{Re} z) u\|^{2}-h\|[P, \widehat{Q}] u\|\|u\|-\mathcal{O}\left(h^{4}\right)\|u\|^{2} \\
\geq & \|(P-\operatorname{Re} z) u\|^{2}-\mathcal{O}\left(h^{2}\right)\|(P-\operatorname{Re} z) u\|\|u\|-\mathcal{O}\left(h^{3}\right)\|u\|^{2} \\
\geq & \|(P-\operatorname{Re} z) u\|^{2}-\mathcal{O}(h)\|(P-\operatorname{Re} z) u\|^{2}-\mathcal{O}\left(h^{3}\right)\|u\|^{2} \\
\geq & \frac{1}{2}\|(P-\operatorname{Re} z) u\|^{2}-\mathcal{O}\left(h^{3}\right)\|u\|^{2},
\end{aligned}
$$


provided that $h$ is small enough. When $z$ is such that $\operatorname{Im} z=\mathcal{O}(h)$ and

$$
\operatorname{dist}\left(\operatorname{Re} z, \cup_{k=1}^{\infty} J_{k}(h)\right) \geq h / C,
$$

for some sufficiently large $C>0$, it follows from (4.3) together with the spectral theorem that

$$
\|(P-\operatorname{Re} z) u\| \geq \frac{h}{C}\|u\|
$$

and using this in (4.5) we obtain, for $h$ small enough,

$$
2\|(\widehat{\mathcal{P}}-z) u\|^{2} \geq \frac{h^{2}}{2 C^{2}}\|u\|^{2}-\mathcal{O}\left(h^{3}\right)\|u\|^{2} \geq \frac{h^{2}}{4 C^{2}}\|u\|^{2}
$$

This immediately implies (4.2) with a new constant $C$. The proof is complete.

Repeating the arguments of Section 3 and using also (2.7), we find that for $\tau$ as in Proposition 4.1, as well as for all $\tau$ with $\operatorname{Im} \tau \geq 2\|a\|_{\infty}+1$, we have

$$
\|R(\tau)\|_{\mathcal{L}\left(H^{\theta}, L^{2}\right)} \leq \frac{\mathcal{O}(1)}{(1+|\tau|)^{1+\theta / 2}}, \quad 0 \leq \theta \leq 2
$$

We now come to prove the propagator expansion. When $k \in \mathbf{N}$ is sufficiently large, we consider the positively oriented contour $\gamma$ given by the following four line segments: $\gamma_{1}=\{x-i ;-k-\alpha / 4-1 / 2 \leq x \leq k+\alpha / 4+1 / 2\}, \gamma_{2}=\{k+\alpha / 4+1 / 2+i y ;-1 \leq$ $\left.y \leq 2\|a\|_{\infty}+1\right\}, \gamma_{3}=\left\{x+i\left(2\|a\|_{\infty}+1\right) ;-k-\alpha / 4-1 / 2 \leq x \leq k+\alpha / 4+1 / 2\right\}$, and $\gamma_{4}=\left\{-k-\alpha / 4-1 / 2+i y ;-1 \leq y \leq 2\|a\|_{\infty}+1\right\}$. Let $f \in H^{\theta}, \theta>0$. An application of the residue theorem allows us to write

$$
\begin{gathered}
\int_{\gamma_{1}} e^{i t \tau} R(\tau) f d \tau=\sum_{\tau \in \operatorname{Spec}(\mathcal{A}),|\operatorname{Re} \tau|<k+1 / 2+\alpha / 4} e^{i t \tau} p_{\tau}(t) f \\
-\int_{\gamma_{2} \cup \gamma_{4}} e^{i t \tau} R(\tau) f d \tau-\int_{\gamma_{3}} e^{i t \tau} R(\tau) f d \tau .
\end{gathered}
$$

Here $p_{\tau}$ is a polynomial in $t$ with values in $\mathcal{L}\left(L^{2}, H^{s}\right)$, for any $s \in \mathbf{R}$. The $L^{2}$-norm of the integral along the segment $\gamma_{2}$ does not exceed

$$
\int_{-1}^{2\|a\|_{\infty}+1} e^{-t y} \frac{\mathcal{O}(1)\|f\|_{H^{\theta}}}{(1+|k|)^{1+\theta / 2}} d y
$$

which tends to zero as $k \rightarrow \infty$. Similarly it follows that the contribution coming from the segment $\gamma_{4}$ vanishes as $k \rightarrow \infty$. When treating the contribution coming from the segment $\gamma_{3}$, now with $k=\infty$, we notice that in view of (4.6) and since $\theta>0$, the contour $\gamma_{3}: \operatorname{Im} \tau=2\|a\|_{\infty}+1$ can be replaced by $\operatorname{Im} \tau=C$ for any $C \geq 2\|a\|_{\infty}+1$. Letting $C \rightarrow \infty$, we conclude that the corresponding contribution to the integral vanishes. We get with $k_{0} \gg 1$,

$$
U(t) f=\sum_{\tau \in \operatorname{Spec}(\mathcal{A}),|\operatorname{Re} \tau|<k_{0}+\alpha / 4} e^{i t \tau} p_{\tau}(t)+\sum_{k=k_{0}}^{\infty} U_{k}(t) f, \quad \text { when } t>0, \quad f \in H^{\theta}
$$


where

$$
U_{k}(t)=\sum_{\tau \in \operatorname{Spec}(\mathcal{A}), \tau \in I_{k}} e^{i t \tau} p_{\tau, k}(t)+\sum_{\tau \in \operatorname{Spec}(\mathcal{A}),-\bar{\tau} \in I_{k}} e^{i t \tau} p_{\tau, k}(t),
$$

and $p_{\tau, k}(t)$ is a polynomial in $t$ with values in $\mathcal{L}\left(L^{2}, H^{s}\right)$, for any $s \in \mathbf{R}$. An application of (4.6) together with the residue theorem shows that for each fixed $t$ we have

$$
\left\|U_{k}(t)\right\|_{\mathcal{L}\left(H^{\theta}, L^{2}\right)}=\mathcal{O}(1) e^{t} \frac{1}{|k|^{1+\theta / 2}}
$$

The absolute convergence of the series $(4.7)$ in $\mathcal{L}\left(H^{\theta}, L^{2}\right), \theta>0$, follows, and this concludes the proof of Theorem 1.4.

5. Proof of Theorem 1.3. As in Section 1, we consider the operator

$$
\mathcal{P}=P+i h Q(z), \quad P=-h^{2} \Delta, \quad Q(z)=2 a(x) \sqrt{z}, \quad z \in[\alpha, \beta]+i[-\gamma, \gamma],
$$

for $0<\alpha<1<\beta, \gamma>0$. In the following discussion, we shall assume that the $H_{p^{-}}$ flow possesses a simple closed trajectory $\gamma:[0, T] \rightarrow p^{-1}(1)$, which is nondegenerate elliptic and such that

$$
\pi(\gamma([0, T])) \cap \operatorname{supp}(a)=\emptyset .
$$

Here $\pi: T^{*} M \rightarrow M$ is the natural projection. Moreover, we shall assume that the linearized Poincaré map $P_{\gamma}$ of $\gamma$ is $N$-elementary, for some $N \geq 4$. We shall establish the existence of a sequence of eigenvalues of the operator $\mathcal{A}$ converging rapidly to the real axis.

Under these assumptions on $\gamma$, it is proved in [6] that there exist quasimodes for $P=P(h)$ associated with $\gamma$ and having polynomially small errors. To be precise, it follows from the results of [6] that there exists a set $H \subset(0,1]$ with $0 \in \bar{H}$, the closure of $H$, and $0<a_{0} \leq a(h) \leq b(h) \leq b_{0}<\infty$, two functions on $H$, such that for any $h \in H$ there exists $m(h) \in \mathbf{N}, E_{j}(h) \in[a(h), b(h)]$, and $u_{j}(h) \in L^{2}, j=1, \ldots m(h)$, such that

$$
\left(P(h)-E_{j}(h)\right) u_{j}(h)=R_{0}(h) \quad \text { in } L^{2}, j=1, \ldots, m(h),
$$

and $\left(u_{j}(h) \mid u_{k}(h)\right)=\delta_{j k}+R_{0}(h)$, where $R_{0}(h)=\mathcal{O}\left(h^{\infty}\right)$. Furthermore, it follows from the construction of [6] that if $A \in \operatorname{Op}_{h}\left(S^{0}\right)$ is such that $W F_{h}(A)$ is contained in a small neighborhood of $\gamma([0, T])$ and $A=I$ microlocally near $\gamma([0, T])$ then

$$
(I-A) u_{j}(h)=\mathcal{O}\left(h^{\infty}\right) \quad \text { in } L^{2}, j=1, \ldots m(h) .
$$

From the assumption (5.1) we infer therefore that

$$
\left(\mathcal{P}-E_{j}(h)\right) u_{j}(h)=R(h), \quad j=1, \ldots m(h) .
$$

where $R(h)=\mathcal{O}\left(h^{\infty}\right)$ in $L^{2}$.

REMARK. The construction of [6] gives quasimodes of $P$ associated with a family of KAM tori of the Poincare mapping, near the closed trajectory $\gamma$. For analytic manifolds, the construction of [6] produces quasimodes with exponentially small errors. We may also recall that under the stronger assumption of the $N$-elementarity of $P_{\gamma}$ for every $N \in \mathbf{N}$, the existence of quasimodes with polynomially small errors can be 
established also by the method of Gaussian beams - see [19] and the references given there. While the construction of [6] gives quasimodes of positive mass, given by the volume of the union of the KAM tori, it is known that the quasimodes obtained by the method of Gaussian beams have mass zero - see [7].

For future reference, we notice that for $z \in[\alpha, \beta]+i[-\gamma, \gamma]$, we have $\operatorname{Im}(\mathcal{P} u \mid u) \geq 0$, and therefore

$$
\left\|(\mathcal{P}-z)^{-1}\right\|_{\mathcal{L}\left(L^{2}, L^{2}\right)} \leq \frac{1}{|\operatorname{Im} z|}, \quad \operatorname{Im} z<0 .
$$

The following result implies Theorem 1.3.

THEOREM 5.1. For any positive function $S(h)$ with $D e^{-D / h}<S(h)=\mathcal{O}\left(h^{\infty}\right)$, for some $D>0$, and such that $S(h) \gg R(h)$, and every $k \in \mathbf{N}$, there exists $h(S, k)>0$ such that for $H \ni h \leq h(S, k)$, the spectrum of $\mathcal{P}=\mathcal{P}(h)$ intersects the set

$$
\left[E_{j}(h)-6 h^{k}, E_{j}(h)+6 h^{k}\right]+i\left[0,2 S(h) h^{-n-1}\right], \quad j=1, \ldots m(h) .
$$

When proving Theorem 5.1, we shall start by establishing the following result, which gives an a priori exponential estimate of the resolvent of $\mathcal{P}$, in a set of size $\sim h$, outside the union of small neigbourhoods of the eigenvalues.

Proposition 5.2. Let $E \in\left[\alpha+1 / C_{0}, \beta-1 / C_{0}\right]$ for some $C_{0}>1$. For every $L>0$ there exists $A>0$ and $h_{0}>0$ such that for $0<h<h_{0}$ we have

$$
\left\|(\mathcal{P}-z)^{-1}\right\|_{\mathcal{L}\left(L^{2}, L^{2}\right)} \leq \frac{A}{h} \exp \left(A h^{1-n} \log \frac{h}{g(h)}\right),
$$

for $|z-E|<L h, \operatorname{dist}(z, \operatorname{Spec}(\mathcal{P})) \geq g(h)$. Here $0<g(h) \ll h$.

Proof. We shall use the idea of [17] and [22] of exploiting a finite rank perturbation to create a gap in the spectrum of $P$ around $E$. Recall from [22] that for any $C>0$ there exists a selfadjoint operator $\widetilde{P}=P+K$, with the same domain as $P$, such that

$$
[E-C h, E+C h] \cap \operatorname{Spec}(\widetilde{P})=0,
$$

and

$$
\|K\|_{\mathcal{L}\left(L^{2}, L^{2}\right)} \leq C h, \quad\|K\|_{\text {tr }} \leq \widetilde{C}(C) h^{2-n}
$$

Here $\|\cdot\|_{\text {tr }}$ denotes the trace class norm. We then introduce

$$
\widetilde{\mathcal{P}}=\widetilde{P}+i h Q(z)
$$

and writing

$$
\widetilde{\mathcal{P}}-z=(\widetilde{P}-z)\left(I+i h(\widetilde{P}-z)^{-1} Q\right),
$$

we see that

$$
(\widetilde{\mathcal{P}}-z)^{-1}=\frac{\mathcal{O}(1)}{h} \quad \text { in } \quad \mathcal{L}\left(L^{2}, L^{2}\right)
$$


when $z$ is such that $|\operatorname{Re} z-E| \leq L h$, provided that $C$ is large enough. Now we write

$$
\mathcal{P}-z=(\widetilde{\mathcal{P}}-z)\left(I-(\widetilde{\mathcal{P}}-z)^{-1} K\right),
$$

and let us introduce

$$
D(z)=\operatorname{det}\left(I-(\widetilde{\mathcal{P}}-z)^{-1} K\right),
$$

which is a holomorphic function in the open disc $D(E, L h)=\{z \in \mathbf{C} ;|z-E|<L h\}$, whose zeros are precisely the eigenvalues of $\mathcal{P}$ in this set. Since the trace class norm of $(\widetilde{\mathcal{P}}-z)^{-1} K$ is $\mathcal{O}(1) h^{1-n}$, we get

$$
|D(z)| \leq \exp \left(\mathcal{O}(1) h^{1-n}\right), \quad z \in D(E, L h) .
$$

An application of Theorem 5.1 from Chapter 5 of [11] shows that when $|\operatorname{Re} z-E| \leq$ $L h$,

$$
\left\|\left(I-(\widetilde{\mathcal{P}}-z)^{-1} K\right)^{-1}\right\|_{\mathcal{L}\left(L^{2}, L^{2}\right)} \leq \frac{\operatorname{det}\left(I+\left|(\widetilde{\mathcal{P}}-z)^{-1} K\right|\right)}{|D(z)|} \leq \frac{e^{\mathcal{O}(1) h^{1-n}}}{|D(z)|},
$$

and when deriving bounds on the resolvent of $\mathcal{P}$, we shall therefore have to estimate $|D(z)|$ from below, away from its zeros. In doing so we remark that when $|z-E|<L h$ and $|\operatorname{Im} z| \geq \mathcal{O}(1) h$ then

$$
(\mathcal{P}-z)^{-1}=\frac{\mathcal{O}(1)}{h}: L^{2} \rightarrow L^{2},
$$

and from (5.6) we get that in this set,

$$
\left(I-(\widetilde{\mathcal{P}}-z)^{-1} K\right)^{-1}=(\mathcal{P}-z)^{-1}(\widetilde{\mathcal{P}}-z)=I+(\mathcal{P}-z)^{-1} K
$$

with

$$
\left\|(\mathcal{P}-z)^{-1} K\right\|_{\text {tr }} \leq \mathcal{O}(1) h^{1-n} .
$$

It follows that

$$
|D(z)| \geq e^{-\mathcal{O}(1) h^{1-n}}, \quad z \in D(E, L h), \quad|\operatorname{Im} z| \geq \mathcal{O}(1) h .
$$

Let now $z_{1}, \ldots z_{N}$ be the zeros of $D(z)$ in $D(E, L h)$. From [22] we recall that $N=$ $\mathcal{O}(1) h^{1-n}$. We factorize

$$
D(z)=G(z) B(z), \quad z \in D(E, L h),
$$

where $G$ is holomorphic and non-vanishing in $D(E, L h)$, and

$$
B(z)=\prod_{j=1}^{N} b_{z_{j}}(z)
$$

is the Blaschke product on $D(E, L h)$, so that

$$
b_{z_{j}}(z)=\hat{b}_{\frac{z_{j}-E}{L h}}\left(\frac{z-E}{L h}\right)=\frac{z-z_{j}}{L h\left(1-\left(\frac{z-E}{L h}\right)\left(\frac{\overline{z_{j}}-E}{L h}\right)\right)} .
$$


Here

$$
\hat{b}_{w}(z)=\frac{z-w}{1-\bar{w} z}, \quad|z| \leq 1,|w|<1,
$$

stands for the standard Blaschke factor for the unit disc. An application of basic factorization theorems in the Banach algebra of bounded holomorphic functions in a disc, or simply using the maximum principle together with (5.7), allows us to conclude that

$$
|G(z)| \leq e^{\mathcal{O}(1) h^{1-n}} \quad \text { in } D(E, L h) .
$$

Taking (5.9) into account we find that for $|\operatorname{Im} z| \geq \mathcal{O}(1) h$,

$$
|G(z)| \geq e^{-\mathcal{O}(1) h^{1-n}},
$$

An application of the Harnack inequality to the harmonic function $\log |G(z)|$, in the form given in Chapter 1 of [17], shows that

$$
|\log | G(z)|| \leq \mathcal{O}(1) h^{1-n}, \quad \text { in } D(E, L h),
$$

after decreasing $L$ slightly. It is easy to see that

$$
|B(z)| \geq e^{-\mathcal{O}(1) h^{1-n} \log \frac{h}{g(h)}}, \quad z \in D(E, L h), \quad \operatorname{dist}(z, \operatorname{Spec}(\mathcal{P})) \geq g(h),
$$

and therefore for such $z$ 's we get,

$$
|D(z)| \geq e^{-\mathcal{O}(1) h^{1-n} \log \frac{h}{g(h)}} .
$$

Combining (5.6) and (5.5) with (5.8) and (5.11), we complete the proof of (5.4).

REMARK. The resolvent bound of Proposition 5.2 together with its proof is closely related to the abstract resolvent estimates for weak non-selfadjoint perturbations of selfadjoint operators, developed in Chapter 1 of [17]. See also [5] and [10] for further references and applications of similar bounds in the theory of resonances and semiclassical pseudospectral theory.

REMARK. For the following argument, it would have been sufficient to derive an exponential bound on $(\mathcal{P}-z)^{-1}$ in a bounded $h$-independent set, rather than in a set of size $\sim h$. Indeed, if $\Omega \subset \subset\{z \in \mathbf{C} ; \operatorname{Re} z>0\}$ is a bounded open $h$-independent neighbourhood of some $E>0$, then a straightforward adaptation of the proof of Proposition 5.2 shows that for all $h$ small enough and $z \in \Omega$, we have

$$
\begin{aligned}
\left\|(\mathcal{P}-z)^{-1}\right\|_{\mathcal{L}\left(L^{2}, L^{2}\right)} & \leq \mathcal{O}(1) \exp \left(\mathcal{O}(1) h^{-n} \log (1 / g(h))\right), \\
\operatorname{dist}(z, \operatorname{Spec}(\mathcal{P})) & \geq g(h)>0 .
\end{aligned}
$$

See also Lemma 6.1 of [10]. The exponent $h^{-n}$ in (5.12) reflects the fact that the number of eigenvalues of $\mathcal{P}$ in $\Omega$ is $\mathcal{O}\left(h^{-n}\right)$ - see [22] for the much more precise result giving the Weyl asymptotics. It seemed to us nevertheless worthwhile, and indeed, of independent interest, to obtain a sharper result in the smaller set, whose size is dictated by the strength of the non-selfadjoint perturbation. Notice also that in the one-dimensional case, $n=1$, Proposition 5.2 gives a polynomial bound on the resolvent of $\mathcal{P}$. It follows that in this case, only eigenvalues can produce a superpolynomial growth of the resolvent. 
We now come to prove Theorem 5.1. When doing so, we follow the argument of [25] and argue by contradiction. Theorem 5.1 is then obtained by applying the argument of [25] as it stands, since an application of the semiclassical maximum principle, Lemma 2 of [25], to $(\mathcal{P}-z)^{-1}$ is now legitimate, in view of Proposition 5.2, (5.12), and the bound (5.2). The proof of Theorem 5.1 is complete.

REMARK. Following [23], it is possible to sharpen the statement of Theorem 5.1 and to estimate the number of eigenvalues of $\mathcal{P}$ in the set

$$
\left[a(h)-\mathcal{O}(1) h^{k}, b(h)+\mathcal{O}(1) h^{k}\right]+i\left[0,2 S(h) h^{-n-1}\right], \quad k \in \mathbf{N} .
$$

Below we shall indicate how to do so, by adapting the argument of [23] to the present setting. To this end, let us introduce the operator

$$
\mathcal{A}(h)=\left(\begin{array}{cc}
0 & 1 \\
-h^{2} \Delta & 2 i a h
\end{array}\right)=\left(\begin{array}{ll}
1 & 0 \\
0 & h
\end{array}\right) h \mathcal{A}\left(\begin{array}{cc}
1 & 0 \\
0 & h
\end{array}\right)^{-1}: H^{1} \times L^{2} \rightarrow H^{1} \times L^{2} .
$$

It is then true that $z \in \operatorname{Spec}(\mathcal{P})$ if and only if $\lambda=\sqrt{z} \in \operatorname{Spec}(\mathcal{A}(h))$. Using the factorization in (5.14) together with Proposition 2.1, we verify that

$$
\left\|(\mathcal{A}(h)-\lambda)^{-1}\right\|_{\mathcal{L}(\mathcal{H}, \mathcal{H})} \leq \frac{\mathcal{O}(1)}{h|\operatorname{Im} \lambda|}, \quad \operatorname{Im} \lambda<0
$$

Here $\mathcal{H}=H^{1} \times L^{2}$, and we equip $H^{1}$ with the corresponding $h$-dependent norm. It is also easy to see, using Proposition 5.2, that the resolvent of $\mathcal{A}(h)$ enjoys an exponential type bound of the same form as $(\mathcal{P}-z)^{-1}$, when away from the eigenvalues. If we now set

$$
U_{j}(h)=\left(\begin{array}{c}
u_{j}(h) \\
E_{j}^{1 / 2}(h) u_{j}(h)
\end{array}\right) \in \mathcal{H}, \quad j=1, \ldots m(h),
$$

then a simple computation shows that

$$
\left(\mathcal{A}(h)-E_{j}^{1 / 2}(h)\right) U_{j}(h)=R(h) \quad \text { in } \mathcal{H},
$$

and $\left(U_{j}(h) \mid U_{k}(h)\right)_{\mathcal{H}}=\left(1+E_{j}(h)+E_{k}(h)\right) \delta_{j k}+R(h)$, where $R(h)=\mathcal{O}\left(h^{\infty}\right)$. Here we have used that the modes $u_{j}(h)$ are orthonormal in $L^{2}$ and ortogonal in $H^{1}$, modulo an error which is $\mathcal{O}\left(h^{\infty}\right)$.

An inspection of [23] combined with the preceding remarks shows that the counting argument of Theorem 1 of [23] applies to the operator-valued function $h(\mathcal{A}(h)-$ $\lambda)^{-1}$ without any change, and demonstrates that for any positive function $S(h)$ such that $\max \left(D e^{-D / h}, h^{-n-2} R(h)\right) \leq S(h)=\mathcal{O}\left(h^{\infty}\right), D>0$, and any $k \in \mathbf{N}$, the rectangle

$$
\left[a(h)^{1 / 2}-6 h^{k}, b(h)+6 h^{k}\right]+i\left[0,2 S(h) h^{-n-1}\right]
$$

contains $\geq m(h)$ eigenvalues of $\mathcal{A}(h)$, for $h$ small enough. It follows that the set (5.13) contains at least $m(h)$ eigenvalues of $\mathcal{P}$. 


\section{REFERENCES}

[1] M. Agranovich, B. Katsenelenbaum, A. Sivov, and N. Voitovich, Generalized method of eigenoscillations in diffraction theory, Wiley-VCH Verlag, Berlin, 1999.

[2] M. Asch And G. Lebeau, The spectrum of the damped wave equation, preprint, Orsay, 1999. See http://www.math.u-psud.fr/ biblio/rt/1999/.

[3] A. Besse, Manifolds all of whose geodesics are closed, Springer Verlag, 1978.

[4] N. BuRQ, Lower bounds for shape resonances widths for long range Schrödinger operators, Amer. J. Math., 124 (2002), pp. 677-735.

[5] N. Burq ANd M. Zworski, Resonance expansions in semi-classical propagation, Comm. Math. Phys., 223 (2001), pp. 1-12.

[6] F. CARdoso AND G. Popov, Quasimodes with exponentially small errors associated with elliptic periodis rays, Asymptot. Analysis, 30 (2002), pp. 217-247.

[7] Y. Colin DE Verdiere, Quasi-modes sur les variétés riemanniennes, Inv. Math., 43 (1977), pp. $15-52$.

[8] S. Cox And E. Zuazua, The rate at which the energy decays in a damped string, Comm. P.D.E., 19 (1993), pp. 213-243.

[9] E. B. Davies, One-parameter semigroups, Academic Press, Inc., London-New York, 1980.

[10] N. Dencker, J. Sjöstrand, And M. Zworski, Pseudospectra of semi-classical (pseudo)differential operators, Comm. Pure Appl. Math., 57 (2004), pp. 384-415.

[11] I. C. Gokhberg And M. G. Krein, Introduction to the theory of linear nonselfadjoint operators, American Mathematical Society, Providence, R.I. 1969.

[12] M. Hitrik, Eigenfrequencies for damped wave equations on Zoll manifolds, Asymptot. Analysis, 31 (2002), pp. 265-277.

[13] M. Hitrik, Expansions and eigenfrequencies for damped wave equations, Journées "Equations aux Dérivées Partielles" (Plestin-les-Grèves 2001), Exp. no VI, Univ. Nantes, 2001.

[14] M. Hitrik And J. SJöstrand, Non-selfadjoint perturbations of selfadjoint operators in 2 dimensions I., Ann. Henri Poincaré, to appear.

[15] L. Hörmander, The analysis of linear partial differential operators III-IV, Springer Verlag 1985.

[16] G. Lebeau, Equation des ondes amorties. Algebraic and geometric methods in mathematical physics (Kaciveli 1993), pp. 73-109, Math. Phys. Stud., 19, Kluwer Acad. Publ., Dordrecht, 1996.

[17] A. S. Markus, Introduction to the spectral theory of polynomial operator pencils, Stiintsa, Kishinev 1986 (Russian). Engl. transl. in Transl. Math. Monographs, 71 Amer. Math. Soc., Providence 1988.

[18] C. Morawetz, Decay for solutions of the exterior problem for the wave equation, Comm. Pure Appl. Math., 28 (1975), pp. 229-264.

[19] J. RaLston, On the construction of quasimodes associated with stable periodic orbits, Comm. Math. Phys., 51 (1976), pp. 219-242.

[20] J. RAUCh AND M. TAYLOR, Decay of solutions to nondissipative hyperbolic systems on compact manifolds, Comm. Pure Appl. Math., 28 (1975), pp. 501-523.

[21] M. Shubova, Riesz basis property of root vectors of nonselfadjoint operators generated by radial damped wave equations, Adv. Diff. Equations, 5 (2000), pp. 623-656.

[22] J. SJöstrand, Asymptotic distribution of eigenfrequencies for damped wave equations. Publ. R.I.M.S., 36 (2000), pp. 573-611.

[23] P. Stefanov, Quasimodes and resonances : sharp lower bounds, Duke Math. J., 99 (1999), pp. $75-92$.

[24] P. Stefanov and G. Vodev, Neumann resonances in linear elasticity for an arbitrary body, Comm. Math. Phys., 176 (1996), pp. 645-659.

[25] S. H. Tang And M. Zworski, From quasimodes to resonances, Math. Res. Lett., 5 (1998), pp. 261-272.

[26] S. H. Tang And M. Zworski, Resonance expansions of scattered waves, Comm. Pure Appl. Math., 53 (2000), pp. 1305-1334.

[27] A. Weinstein, Asymptotics of eigenvalue clusters for the Laplacian plus a potential, Duke Math. J., 44 (1977), pp. 883-892.

[28] M. Zworski, Resonance expansions in wave propagation, Séminaire E.D.P., 1999-2000, École Polytechnique, XXII-1-XXII-9. 